\title{
Propuesta De Modelo De Gestión De Información Y Conocimiento En El Marco De Un Observatorio Turístico Cantonal En La Ciudad De Riobamba
}

\author{
Diana Paola Salazar Andrade \\ Docente - Investigadora de la Universidad Técnica de Cotopaxi \\ Extensión La Maná \\ Catalina Margarita Verdugo Bernal \\ Docente - Investigadora de la Facultad de Recursos Naturales \\ Escuela Superior Politécnica de Chimborazo - Ecuador \\ Natalia Geoconda Zambrano Cuadro \\ Docente - Investigadora de la Universidad Técnica de Cotopaxi \\ Extensión La Maná \\ Otto Fernando Balseca \\ Docente - Investigador de la Facultad de Mecánica \\ Escuela Superior Politécnica de Chimborazo - Ecuador \\ Mayra Elizabeth Cáceres Mena \\ Docente - Investigadora de la Facultad de Recursos Naturales \\ Escuela Superior Politécnica de Chimborazo - Ecuador \\ Oscar Iván Granizo Paredes \\ Sub-Director Posgrado-ESPOCH
}

doi: 10.19044/esj.2016.v13n2p51 URL:http://dx.doi.org/10.19044/esj.2016.v13n2p51

\begin{abstract}
We present a sustainable management model capable of delivering timely products and services demanded by public and private tourism stakeholders. The case study is for the strategic decision-making of the tourism sector in Riobamba County, Ecuador, within the framework of a Tourism Observatory as a means of information management.

Other cases of information management related to tourism and literature on national and international tourist observatories were analyzed. Locally, information gaps were identified through personal interviews with actors in the sector.

The preferred organizational structure follows the Red Model, which uses the construction of knowledge as a barometer of quality, an opportunity for investment, market research, learning spaces and exchange of experiences. It also presents the profile of services and products that will be offered by the
\end{abstract}


observatory, flow of processes, financing alternatives and strategic alliances for its operation.

Our research demonstrates the willingness of tourism stakeholders to move from information management to knowledge management. It is concluded that our Information and Knowledge Management Model could be promoted in the future as an alternative to address real problems in information management.

We recommend implementing our Information and Knowledge Management Model because it demonstrates a contribution to the generation of unique and transferable knowledge among tourism stakeholders. It is indispensable for stakeholder institutions to work in coordination with joint responsibility for the success of the Tourism Observatory.

Keywords: Management Model, Information, Knowledge, Observatory, Tourism

\section{Resumen}

La investigación tuvo como propósito analizar un modelo de gestión sustentable, capaz de entregar productos y servicios oportunos demandados por los actores clave del turismo públicos y privados para la toma de decisiones estratégicas del sector en el cantón de Riobamba, en el marco de un Observatorio Turístico, como alternativa para abordar la problemática de manejo de información.

Se analizaron casos de manejo de información y conocimiento de observatorios turísticos nacionales e internacionales, se identificaron carencias de información turística a través de entrevistas personales a actores locales.

Los resultados obtenidos fueron la estructura organizativa del Modelo Red y los procesos para la construcción del conocimiento como barómetro de la calidad, portal de oportunidad de inversión, sondeo de mercados, espacios de aprendizaje e intercambio de experiencias. Se presenta además el perfil de servicios y productos que serán ofertados por el observatorio, flujograma de procesos, alternativas de financiamiento y alianzas estratégicas básicas para su funcionamiento.

La investigación realizada evidencia la voluntad explícita de los actores del turismo de ir de la gestión de información a la gestión del conocimiento por lo cual se concluye que el Modelo de Gestión de Información y Conocimiento podría constituirse en el tiempo, como alternativa para abordar la problemática actual en el manejo de información.

Se recomienda implementar el modelo de gestión propuesto que contribuya a la generación de conocimiento único y transferible entre los actores 
turísticos, para lo cual es indispensable trabajar en coordinación institucional con corresponsabilidad para el éxito del Observatorio Turístico.

Palabras-clave: Modelo, Gestión, Información, Conocimiento, Observatorio, Turismo

\section{Introducción}

La creciente actividad económica del turismo ${ }^{6}$ es un área con cobertura informativa limitada, existen algunos datos pero en muchos casos es información desactualizada sin un sistema de obtención, análisis y publicación de información que integre diferentes variables y genere conocimiento de utilidad para orientar la toma de decisiones (Informe Anual de la OMT. 2015: 16).

Algunos territorios de afluencia turística que han detectado la necesidad de recolectar y procesar información, han abordado la idea de diseñar un observatorio turístico, como una herramienta de investigación y monitoreo de estadísticas de turismo para posteriormente difundir la información entre los diferentes actores. (Instituto de Competitividad Turística, 2015).

Misma situación de carencia de información (Ortega y Martinez. 2005: 172) ocurre en el cantón Riobamba, es escasa y no del nivel de detalle que el sector requiere, si se compara con la abundancia existente en otras áreas de la economía, por la ausencia de una metodología de operación que aborde esta problemática entre los actores del turismo, que, aumente la confiabilidad disminuya la incertidumbre y evite la afectación en la calidad de los productos y servicios turísticos (Turmero, 2011:14).

En este contexto se propone un modelo de gestión de la información y conocimiento, bajo el concepto de un Observatorio Turístico Cantonal (Santágata, 2011:15) como alternativa para solucionar la problemática identificada en el acceso a la información y a la ausencia de conocimiento del sector. Esto con la finalidad de dar lineamientos de ¿cómo estructurar el manejo de información y conocimiento en un Observatorio Turístico, a fin de asegurar su adecuación y oportunidad? (Metodología par definición de indicadores turísticos Nayarit, 2006:2)

El modelo de gestión propenderá que el levantamiento, procesamiento y análisis de la información sea relevante, permanente y sistemático asegurando la generación de conocimiento útil y confiable para la acertada toma de acciones por parte de actores del turismo local que

${ }^{6}$ Con más de un billón de turistas viajando por el mundo cada año, el turismo se ha convertido en uno de los sectores líderes de la economía, contribuyendo el 10\% del GDP global y un 6\% del total de exportaciones mundiales (Reporte anual de la OMT. 2015). 
encaminen al desarrollo turístico sostenible del cantón Riobamba (Gobierno Autónomo Descentralizado del Municipio de Riobamba. 2015).

\section{Metodología}

La presente investigación se desarrolló mediante el método comparativo, cualitativo y analítico en las siguientes etapas:

Para lo cual se analizó casos de manejo de información y conocimiento de observatorios turísticos nacionales e internacionales, además se identificaron carencias de información turística a través de entrevistas personales a actores locales, que permitió definir el modelo más idóneo para el manejo del observatorio.

Precisar cómo se genera conocimiento entre los actores del turismo es un proceso de articulación, trabajo en equipo, especialización y análisis de criterios expertos que podrían estar enfocados en la propuesta de un Modelo Red de Gestión de Información y Conocimiento para el Observatorio Turístico que garantizaría corresponsabilidad institucional, participación y aportación de financiamiento. Este modelo por su grado de autonomía puede generar sostenibilidad financiera, resultados positivos, confiables y útiles sin sujeción a presiones de grupos políticos.

\section{Analizar casos destacados de manejo de información en casos relevantes de observatorios turísticos, a nivel nacional e internacional.}

El estudio del arte permitió analizar casos destacados de manejo de información en casos relevantes de observatorios turísticos, a nivel nacional e internacional a través del método comparativo mediante una matriz tomando como referencia los casos Perú y Galápagos.

Con la matriz comparativa se analizó variables como:

$\checkmark$ Marco institucional: estructura directiva pública, privada o mixta

$\checkmark$ Vínculo con actores turístico

$\checkmark$ Productos y servicios que el observatorio genera

$\checkmark$ Esquema de manejo de información y conocimiento

$\checkmark$ Periodicidad de la información

$\checkmark$ Forma de presentación de resultados

$\checkmark$ Temas destacados en el sitio web (si cuentan con pág. web).

Este ejercicio permitió identificar los aspectos de mayor relevancia para los actores del turismo en manejo de información y conocimiento por los observatorios analizados, capitalizando las experiencias en la propuesta presente. 
Identificar el perfil de información y conocimiento requeridos por los actores clave de la actividad turística cantonal.

El método cualitativo permitió identificar el perfil de información y conocimiento requeridos por los actores clave de la actividad turística cantonal para lo cual se aplicaron entrevistas personales a expertos en el campo turístico como representantes del MINTUR, Cámara de Turismo, Unidad de Turismo del GAD Municipal y Provincial así como a académicos vinculados a la formación en turismo en lo referente al sector público.

Mientras que en el sector privado se consideró el aporte de prestadores de servicios como Gerentes de Operadoras de Turismo, Agencias de Viajes y administradores de Hoteles de segunda categoría de la ciudad de Riobamba,

Esta investigación permitió identificar necesidades de información, nivel de análisis, frecuencia, percepciones y opiniones frente al modelo de información y conocimiento del Observatorio Turístico Cantonal.

Proponer un modelo de generación y manejo de información y conocimiento, en el marco de un Observatorio turístico cantonal.

A través del método analítico se identificaron estructuras y procesos propuestos para el modelo de generación y manejo de información y conocimiento, en el marco de un Observatorio turístico cantonal, que abarque la estructura y funcionamiento, perfil de productos y servicios. Procesos operacionales, sostenibilidad económica, comunicación y alianzas estratégicas para el funcionamiento del observatorio.

\section{Resultados}

Como hallazgos de la investigación se presenta que en la actualidad Observatorios Turísticos a nivel nacional e internacional juegan un rol importante en el desarrollo sustentable de los territorios. Es por esta razón que en países europeos como España, Francia y Latino americanos como Perú, Chile y Argentina han creado observatorios en los últimos años para orientar la política turística en provincias y regiones.

La mayor parte de observatorios turísticos a nivel nacional e internacional generan reportes estadísticos de la evolución del turismo considerando indicadores como ingreso de turistas, gasto promedio por día, ocupación hotelera, grado de satisfacción del cliente, y muy pocos como el caso Perú trabaja con mayor énfasis en investigaciones turísticas, interpretación de datos, y análisis especializado de la información recopilada. A continuación se presenta la matriz comparativa de los casos analizados: Perú y Galápagos. 
MATRIZ COMPARATIVA DE CASOS DESTACADOS EN MANEJO DE INFORMACIÓN Y

CONOCIMIENTO DE OBSERVATORIOS TURÍSTICOS NACIONALES E INTERNACIONALES

\begin{tabular}{|c|c|c|}
\hline VARIABLES & $\begin{array}{l}\text { OBSERVATORIO TURÍSTICO } \\
\text { NACIONAL DE PERÚ }\end{array}$ & $\begin{array}{l}\text { OBSERVATORIO TURÍSTICO } \\
\text { DE GALÁPAGOS }\end{array}$ \\
\hline $\begin{array}{c}\text { PAÍS DE LA } \\
\text { INICIATIVA DE } \\
\text { O.T. }\end{array}$ & Perú & Ecuador \\
\hline $\begin{array}{c}\text { DISPONIBILIDA } \\
\text { D DE PÁGINA } \\
\text { WEB }\end{array}$ & $\begin{array}{c}\text { http://www.observatorioturisticodelper } \\
\text { u.com/ }\end{array}$ & $\begin{array}{c}\text { http://www.observatoriogalapagos.g } \\
\text { ob.ec/ }\end{array}$ \\
\hline $\begin{array}{c}\text { MARCO } \\
\text { INSTITUCIONAL }\end{array}$ & $\begin{array}{l}\text { Vinculación Universidad San Martín } \\
\text { de Porres/empresa privada. }\end{array}$ & $\begin{array}{l}\text { Iniciativa de carácter público en } \\
\text { funcionamiento bajo resolución de } \\
\text { del Consejo de Gobierno del } \\
\text { Régimen Especial de Galápagos. }\end{array}$ \\
\hline $\begin{array}{l}\text { FINANCIAMIENT } \\
\text { O }\end{array}$ & Privado & Público \\
\hline $\begin{array}{l}\text { FECHA DE } \\
\text { CREACIÓN }\end{array}$ & 2013 & 2011 \\
\hline $\begin{array}{l}\text { ESTRUCTURA } \\
\text { DE TOMA DE } \\
\text { DECISIONES }\end{array}$ & $\begin{array}{l}\text { Provee información precisa, fiable, } \\
\text { constante en el tiempo y comparable, } \\
\text { sobre indicadores turísticos para la } \\
\text { adecuada toma de decisiones, de los } \\
\text { organismos e instituciones públicas y } \\
\text { privadas interesadas en el turismo. }\end{array}$ & $\begin{array}{c}\text { Información generada para utilizar } \\
\text { en proyectos de planificación y } \\
\text { manejo del turismo a nivel local y } \\
\text { regional. } \\
\text { Al analizar la información se } \\
\text { identifican indicadores críticos de } \\
\text { impacto del turismo (tanto positivos } \\
\text { como negativos) para tomar } \\
\text { decisiones a nivel de políticas } \\
\text { públicas, programas y proyectos, y } \\
\text { actividades puntuales. } \\
\text { El OTG es una fuente de } \\
\text { información y de consulta de } \\
\text { actores privados y comunitarios del } \\
\text { turismo local y de los miembros } \\
\text { del Grupo Técnico, así como de } \\
\text { otras instituciones, } \\
\text { universidades e individuos en } \\
\text { general } \\
\text { La base de datos que maneja el O.T. } \\
\text { permite crear productos y análisis } \\
\text { personalizados de acuerdo a las } \\
\text { necesidades de intervención. }\end{array}$ \\
\hline
\end{tabular}

Datos estadísticos sobre la tendencia de la demanda, la oferta turística y

PERFIL DE SERVICIOS Y PRODUCTOS nivel de satisfacción de los turistas. Investigaciones referentes al turismo. Publicación de la Revista Turismo y

Patrimonio en diferentes volúmenes. Imágenes exclusivas de Perú.

Información General: demografía, geografía, distancias, clima y temperatura.

Información Económica Nacional,

\section{Presenta información sobre} indicadores de demanda, oferta, e impactos sociales y ambientales del turismo.

Identifica tendencias de mercado y patrones de desarrollo de la actividad turística en Galápagos. Realiza proyecciones sólidas, confiables, y análisis econométricos.

Integra información generada por 
Regional y Mundial.

Consultas y Asesorías On-line otros sistemas de monitoreo como:

Sistema Integrado de Indicadores de Galápagos del CGREG, Sistema de Manejo de visitantes SIMAVIS y las Tarjetas de control de Tránsito que maneja la DPNG.

\section{PERIODICIDAD DE INFORMACIÓN}

Trimestral y anual.
Semestral, anual

\section{FORMA DE PRESENTACIÓN DE RESULTADOS}

Boletines informativos trimestrales de coyuntura y las memorias anuales a través de la página web.
Informes semestrales y anuales mediante la página web (estadísticas en línea y boletines) que está a disponibilidad de empresas públicas, privadas y público en general. Además del material físico impreso en la oficinas de la Dirección Regional Galápagos del Ministerio de Turismo.

Proyecto con apoyo y asistencia de la WWF, Ministerio de Turismo (MINTUR), la Dirección del Parque Nacional Galápagos (DPNG) y el Consejo de Gobierno del Régimen Especial de Galápagos (CGREG) Prestadores de servicios turísticos facilitan información del número de plazas y el porcentaje de ocupación, personal contratado y género de los mismos.

Turistas facilitan información por medio de encuestas en diferentes puntos turísticos.

Obtiene información primaria del turista, establecimientos turísticos.

Recopila y analiza información mediante 400 encuestas mensuales. Difunde indicadores clave de oferta, demanda, sociales y ambientales para generar conocimiento sobre las islas Galápagos.

Presenta información colateral de carácter académica

Difunde informes de coyuntura y boletines con indicadores de turismo

\section{TRANSFORMACI ÓN DE INFORMACIÓN EN CONOCIMIENTO}

Analizan y evalúa en base a la información cuantitativa el comportamiento macroeconómico del sector turismo en Perú, se transforma en conocimiento cuando da a conocer la creciente importancia económica y posibilidades de desarrollo para los
Compara mensualmente la estacionalidad de los visitantes y el ingreso de visitantes a las Islas Galápagos.

Evalúa el movimiento marítimo inter-islas y el crecimiento intermensual. 
próximos años. (predicciones y/o proyecciones)

El Banco de Datos Turístico, Económico, Estadístico y Financiero, así como del Inventario Turístico es un sistema informático que a más de proveer información de carácter cualitativo y cuantitativo es un agente promotor permanente de investigación en el campo turístico.

Identifica la demanda de información turística y legal de los principales actores sociales del sector turismo en la cual se

Evalúa la realización de actividades de promoción de inversión y de servicios, para la mejora de la competitividad del sector turístico, y la posibilidad de adaptarse rápidamente a la evolución del mercado y a los cambios, tomando decisiones de políticas sectoriales, empresariales y de marketing, basadas en la información y conocimiento generado.

Mide la calidad en la prestación del servicio desde la percepción de los usuarios (porcentaje de satisfacción) y se convierte en conocimiento cuando recomienda medidas de mejora de variables con algún grado de insatisfacción.

Da a conocer la tasa de crecimiento del turismo receptor de Perú y se convierte en conocimiento cuando permite realizar una comparación anual.
Compara en crecimiento económico por el flujo turístico durante los feriados, para próximas inversiones $\mathrm{y}$ oferta de productos y servicios.

En el ámbito social y ambiental únicamente da a conocer información sobre porcentajes e indicadores anuales.

\section{Realizado por: Diana Salazar}

Fuente: García, J. C., Rangel, E., \& Farías, M. A. 2013; Observatorio Turístico del Perú. 2013

Otro hallazgo de la investigación determina que las fuentes actuales de información turística son una base de evaluación del sector, sin embargo no satisface la demanda de los actores. Por lo tanto se evidencia la voluntad explícita de los actores del turismo de ir más allá de la gestión de información, simple, es decir del estudio básico de estadísticas e indicadores convencionales hacia la inmersión en el conocimiento real del sector turístico. Como se ilustra en el siguiente gráfico: 
Gráfico 1: Nivel de investigación de la información requerida por los actores del turismo

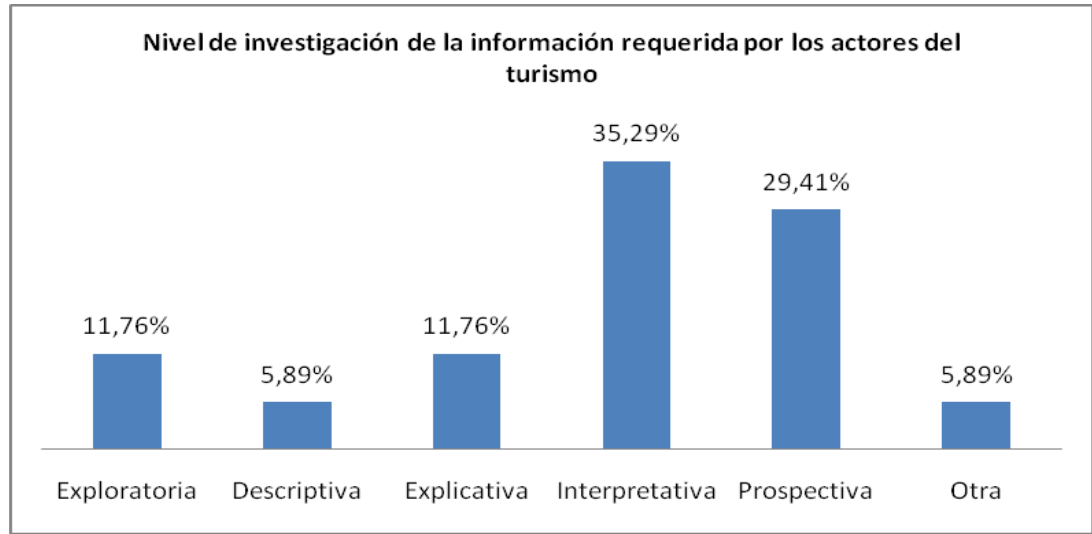

Elaborado: Diana Salazar

Fuente: Trabajo de campo. 2015

Los entrevistados del sector público y privado mostraron gran interés y aceptación, hacia la alternativa propuesta, mencionando que su aporte sería entregar la información que se encuentra a su disposición mayoritariamente, colaborar para la obtención de información en algunos casos y aporte de financiamiento para la realización de estudios de investigación turística en otros.

Finalmente se analizó las ventajas y desventajas de los posibles modelos de gestión para el observatorio turístico y se definió como alternativa más idónea al Modelo Red de Gestión que sea capaz de generar confiabilidad para la inversión turística de empresarios; además debería proveer informes sobre tendencias y expectativas de la demanda a nivel local a partir de la experiencia y conocimiento compartido por líderes turísticos en espacios de diálogo y debate.

Si bien la gestión del conocimiento es un centro inagotable de creatividad, de desarrollo, de competencias personales y organizacionales, de productividad, que para su efectividad requiere articulación institucional, sinergia y corresponsabilidad entre los actores institucionales para generar nuevo conocimiento que oriente la toma de decisiones estratégicas para el desarrollo turístico cantonal. Paro lo cual se ilustra en el siguiente gráfico: 


\section{Modelo de gestión de información y conocimiento Observatorio turístico cantonal}

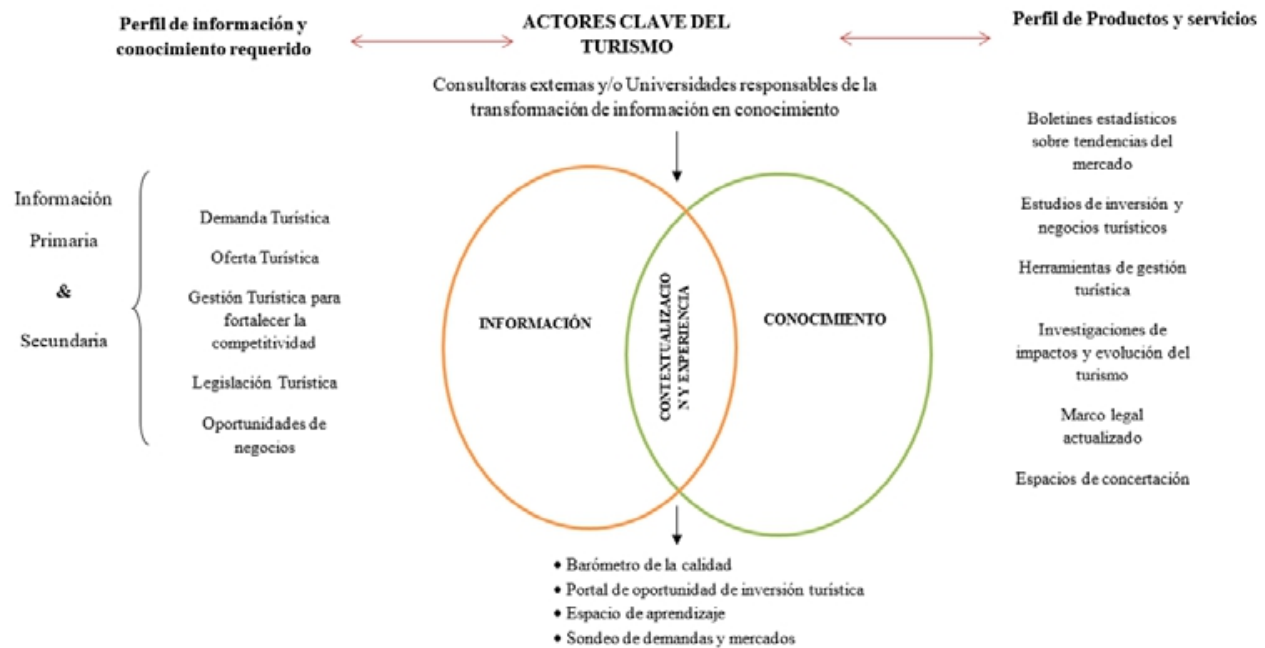

\section{Conclusion}

Los observatorios deben contar con autonomía de gestión para que se consolide su funcionamiento y sus resultados estén deslindados de presiones de grupos políticos o gobiernos de turno. Algunos observatorios son administrados por universidades, empresas mixtas donde se garantiza el aporte público y privado o por la empresa privada como en el caso Perú.

$\checkmark$ El modelo de Gestión de Información y Conocimiento se ha desarrollado de acuerdo a las necesidades del campo turístico, como alternativa para abordar la problemática actual en el manejo de información, generación de conocimiento con valor y accesibilidad, permitiendo identificar oportunidades de inversión turística.

$\checkmark$ La aplicabilidad y sustentabilidad del Modelo Red se determinará con el tiempo de acuerdo a la aportación en la gestión turística, siempre y cuando se consideren ciertas características de participación institucional con responsabilidad bajo convenios con resultados, autonomía de gestión, fuentes de información confiables, equipo humano de alto nivel de especialización que garantice la calidad de productos y servicios.

\section{References:}

1. García, J. C., Rangel, E., \& Farías, M. A. (19 de Junio de 2013). Informe Galápagos 2011-2012. DPNG, GCREG, FCD y GC. Puerto. Recuperado el 3 de Mayo de 2015, de El Observatorio de Turismo de Galápagos: El sistema de monitoreo del: 
http://www.galapagos.org/wp-content/uploads/2013/06/2013-13TOUR-observatory.garcia-rangel-farias.pdf

2. Importancia de la comunicación en los destinos turísticos internacionales: la percepción de los turistas extranjeros en los destinos españoles enrique ortega martínez, beatriz rodríguez herráez. 2005: 172

3. Instituto de Competitividad Turística. (2015). Sectur. Recuperado el 30 de Abril de 2015, de Ictur: http://ictur.sectur.gob.mx/index.php/servicios/centro-dedocumentacion.

4. Metodología para la definición de indicadores en base a un Sistema turístico. Gobierno de

5. Riviera Nayarit. México. 2006. Diapositiva 2.

6. Observatorio Turístico del Perú. (2013). Badatur. Recuperado el 3 de Mayo de 2015, de http://www.observatorioturisticodelperu.com/

7. Quiroga, L. A. (Septiembre-Octubre de 2002). Scielo- Acimed, versión ISSN 1024-9435. Recuperado el 28 de Mayo de 2015, de Gestión de información, gestión del conocimiento y gestión de la calidad en las organizaciones: http://scielo.sld.cu/scielo.php?script=sci_arttext\&pid=S102494352002000500004

8. Santaga, H. 2011. La Importancia de un Observatorio de turístico en Provincia de Buenos Aires como instrumento de orientación en la toma de decisiones y en la planificación. Pp. 15. Notas en Turismo y Economía. Año II. Nro. III. 2011. ISSN 1853-1504

9. Turmero, I. 2013. Sistemas de información turística. Puerto Ordaz. 2013, Pp.14. Calidad de revista científica Española. 